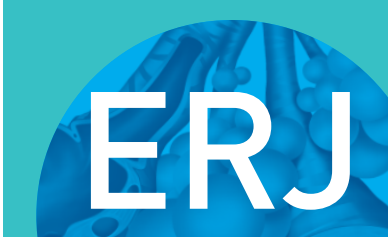

open research

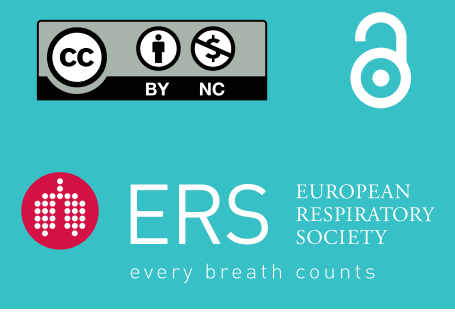

\section{Pubertal onset with adulthood lung function mediated by height growth in adolescence}

\author{
Liang $\mathrm{Li}^{1}$, Hongmei Zhang ${ }^{1}$, John W. Holloway $\mathbb{C}^{2}$, A. John Henderson ${ }^{3 \dagger}$, \\ Susan Ewart ${ }^{4}$, Caroline L. Relton ${ }^{5}$, S. Hasan Arshad ${ }^{6,7}$ and Wilfried Karmaus (1) ${ }^{1}$
}

Affiliations: 'Division of Epidemiology, Biostatistics, and Environmental Health, School of Public Health, University of Memphis, Memphis, TN, USA. ${ }^{2}$ Human Development and Health, Faculty of Medicine, University of Southampton, Southampton, UK. ${ }^{3}$ Population Health Sciences, University of Bristol, Bristol, UK. ${ }^{4}$ College of Veterinary Medicine, Michigan State University, East Lansing, MI, USA. ${ }^{5}$ MRC Integrative Epidemiology Unit, University of Bristol, Bristol, UK. ${ }^{6}$ Clinical and Experimental Sciences, Faculty of Medicine, University of Southampton, Southampton, UK. ${ }^{7}$ The David Hide Asthma and Allergy Research Centre, St Mary's Hospital, Newport, UK.

Correspondence: Hongmei Zhang, Division of Epidemiology, Biostatistics, and Environmental Health, School of Public Health, University of Memphis, 3825 DeSoto Ave, Memphis, TN 38152, USA.

E-mail: hzhang6amemphis.edu

\section{ABSTRACT}

Background: Age of pubertal onset is associated with height and lung function in adulthood. It is unknown whether height growth in adolescence mediates the association of age at puberty with early adult lung function.

Methods: Data from the Isle of Wight (IOW) birth cohort $(n=1261)$ were examined in the study. Ages of pubertal events, height at ages 10 and 18 years and lung function parameters (forced vital capacity (FVC) and forced expiratory volume in $1 \mathrm{~s}\left(\mathrm{FEV}_{1}\right)$ ) at 26 years were included in a path analysis to assess the mediation effects of height growth. Findings were tested in the Avon Longitudinal Study of Parents and Children (ALSPAC) birth cohort.

Results: In females in the IOW cohort, age at menarche and body hair growth showed a positive indirect association with FVC (menarche: indirect effect coefficient (IEC) $=0.13,95 \%$ CI $0.05-0.20, p=1.28 \times 10^{-3}$; body hair growth: IEC $=0.08,95 \%$ CI $0.01-0.15, p=0.017$ ) and $\mathrm{FEV}_{1}$ (menarche: IEC $=0.09,95 \% \mathrm{CI}$ $0.01-0.17, \mathrm{p}=0.028$; body hair growth: IEC $=0.07,95 \% \mathrm{CI} 0.01-0.14, \mathrm{p}=0.043$ ) at 26 years through height growth and lung function at 18 years. In males, age at body hair growth (IEC $=0.08$; $95 \%$ CI $0.01-0.15$, $\mathrm{p}=0.047$ ), growth spurt (IEC=0.09; 95\% CI 0.01-0.17, $\mathrm{p}=0.034)$ and facial hair growth (IEC=0.09; 95\% CI $0.02-0.16, p=0.014)$ had positive indirect effects on FVC at 26 years, but voice deepening did not show statistically significant indirect effects $(\mathrm{p}>0.05)$. For pubertal events available in the ALSPAC cohort, results consistent with the IOW cohort were found for both females and males.

Conclusion: Effects of age of puberty on FVC in early adulthood are likely mediated by height growth during adolescence.

@ERSpublications

Height growth in adolescence mediates the association of age of pubertal onset with FVC in young adults. For females, such mediation effects are also identified for $\mathrm{FEV}_{1}$. https://bit.ly/3mwSTi6

Cite this article as: Li L, Zhang $\mathrm{H}$, Holloway JW, et al. Pubertal onset with adulthood lung function mediated by height growth in adolescence. ERJ Open Res 2020; 6: 00535-2020 [https://doi.org/ 10.1183/23120541.00535-2020].

This article has supplementary material available from openres.ersjournals.com

Received: 28 July 2020 | Accepted after revision: 27 Aug 2020

Copyright $\odot$ ERS 2020. This article is open access and distributed under the terms of the Creative Commons Attribution Non-Commercial Licence 4.0. 


\section{Introduction}

Lung function assessments are often performed to diagnose, monitor and evaluate disease status and health conditions such as asthma [1], COPD [2], infectious respiratory disease [3] and lung cancer [4]. Different lung function parameters, e.g., the spirometry measures forced vital capacity (FVC) and forced expiratory volume in $1 \mathrm{~s}\left(\mathrm{FEV}_{1}\right)$, represent different physiological and clinical conditions [5]. Multiple studies have indicated that distinctive patterns of lung function development have substantial implications for health and disease [6-10]. For example, early below average $\mathrm{FEV}_{1}$ trajectory is associated with increased risk of developing COPD by middle age [8].

Adolescence is an important period that is accompanied by significant sex-dependent changes, e.g., puberty, rapid growth and often body mass index (BMI) increase. It is also a critical period for the maturation of lung function [11]. It has been previously shown that age at menarche (in females), body hair growth (in males) and peak height velocity (in both sexes) are associated with lung function in later life [11-15]. For instance, early menarche is associated with better lung function development in adolescence, but the opposite in adulthood [13]. A recent Mendelian randomisation study suggested that pubertal timing, rather than specific pubertal events, was associated with lung function in both females and males [13].

Age at puberty is also closely related to height growth during adolescence, and this association is potentially sex-dependent. For girls, earlier age at puberty is associated with shorter height, while in boys, earlier age at puberty and slow progression through puberty is linked to taller height in early adulthood [16]. A strong association between height growth during adolescence and lung function in adulthood has been previously observed. Lung function increases with height in adolescence, although the association can be non-linear $[17,18]$. However, after adolescence, lung volume continues to increase after adult height reaches a plateau $[19,20]$. Recent findings in the Avon Longitudinal Study of Parents and Children (ALSPAC) cohort indicated that subjects with greater peak velocity of height growth in puberty had higher FVC and $\mathrm{FEV}_{1}$ in young adulthood [15] and that height growth during adolescence was associated with age of puberty and with lung function at young adulthood. However, it is unknown whether height growth plays a mediating role in the association of age at puberty with lung function in early adulthood.

The objectives of this study were to test whether height growth during adolescence mediated the association of age at puberty with lung function. We applied path analyses [21] using data in the Isle of Wight (IOW) birth cohort established in 1989/1990 in the United Kingdom [22].

\section{Methods}

\section{Study population}

A population-based birth cohort study was initiated in 1989 on the Isle of Wight, UK to prospectively study the natural history of allergic diseases, asthma and lung function and associated risk factors [22]. Of the 1536 children born and recruited in this period, 1261 were available for further follow-up with data collected at ages 10, 18 and 26 years (figure S1). The Local Research Ethics Committee approved this study. Written informed individual or parental consent was obtained at in-person visits. In addition to demographic information and age of pubertal events collected via questionnaire, at ages 10, 18 and 26 years, height and weight were measured, and lung function parameters and allergic conditions were assessed.

\section{Ages of pubertal onset and height growth in adolescence}

At 18 years of age, subjects were interviewed to recall the age of onset for pubertal events using questions relating to pubertal events from the National Institute of Child and Human Development (NICHD) [16]. For females, these included questions on body hair growth, breast growth, menarche, skin changes and growth spurt. Those in males included body hair growth, facial hair growth, voice deepening, skin changes and growth spurt. The detailed measurements of pubertal events are described elsewhere [16].

Height at ages 10 and 18 years was recorded through standard height measurement; in those who did not attend the clinic the information was acquired by self-report. Height growth was baseline-adjusted and calculated as change in height from 10 to 18 years (representing pre- to post-adolescence) divided by the height at 10 years. This measure considers the height gain during adolescence adjusted by pre-adolescence height.

\section{Lung function assessment}

Assessment of lung function at 18 and 26 years of age was conducted by the KoKo spirometry software package on a portable desktop device (PDS instrumentation, Louisville, KY, USA) [23]. Tests were conducted according to the guidelines of the American Thoracic Society and European Respiratory Society [24]. The lung function measurements included in this study were FVC and $\mathrm{FEV}_{1}$. 


\section{Potential confounders}

Factors related to growth, demographic features and environmental exposure might confound association of age of onset of pubertal events with height growth and lung function. In this study, low birth weight status, maternal smoking status during pregnancy, asthma status and BMI at age 10 years, socioeconomic status (SES) and personal smoking status at age 18 years were considered as potential confounders. Birth weight and maternal smoking during pregnancy were obtained at birth of the child. Asthma was defined as having "physician diagnosed asthma" and either "wheezing or whistling in the chest in the last 12 months" and/or "current treatment for asthma", using the International Study of Asthma and Allergies in Childhood (ISAAC) questionnaire [25]. BMI was defined by weight (kilogrammes) divided by the square of height (metres). SES was ascertained by "low", "medium" and "high" according to assessment of level of household income and number of rooms in the house.

\section{Statistical analyses}

Since there are major differences between boys and girls in pubertal events, all data analyses were stratified by sex. To compare samples included in the present study and in the total cohort, one sample t-tests were used on main continuous variables of interest including age of onset of pubertal events, lung function and height at ages 10 and 18 years.

The direct and indirect effects of age at puberty on lung function measurements $\left(\mathrm{FVC}\right.$ and $\left.\mathrm{FEV}_{1}\right)$ at ages 18 and 26 years were examined using path analyses via structural equation modelling $[21,26]$. Height growth was included in the path analyses as a potential mediator (figure 1). For the purpose of selecting pubertal events to be included in the path analyses, three analyses using linear regressions were conducted: the first two analyses examined the association of age of onset for pubertal events with lung function parameters (FVC and $\mathrm{FEV}_{1}$ ) at both ages 18 and 26 years, and the third analysis tested the association of age of onset for pubertal events with height growth. Pubertal events associated with the lung function parameters at one of the two ages (18 and 26 years) and with height growth were included in the subsequent path analyses (figure 1), and we performed a path analysis for each of these pubertal events. Path analyses were performed using PROC CALIS in SAS 9.4 (SAS, Cary, NC, USA). A p-value $<0.05$ was deemed as being statistically significant.

\section{Replication cohort}

Findings in the IOW birth cohort were further tested in the ALSPAC birth cohort, UK [27-29]. ALSPAC is a multi-generational prospective birth cohort study investigating influences on health and development across the life course [27-29]. All pregnant women residing in and around the city of Bristol (south-west UK) during 1990-1992 were eligible to enrol in the cohort, and 14062 live newborns were recruited. Data on demographics, ages of puberty onset (menarche and body hair growth for females, and body hair growth for males), height at ages 10 and 17 years, along with lung function parameters $\mathrm{FEV}_{1}$ and FVC measured at ages 15/17 and 24 years were included in our study. Details of all the data are available on the study website (www.bristol.ac.uk/alspac/researchers/our-data/). Ethical approval for the study was obtained from the ALSPAC Ethics and LAW Committee and the Local Research Ethics Committees. Height growth

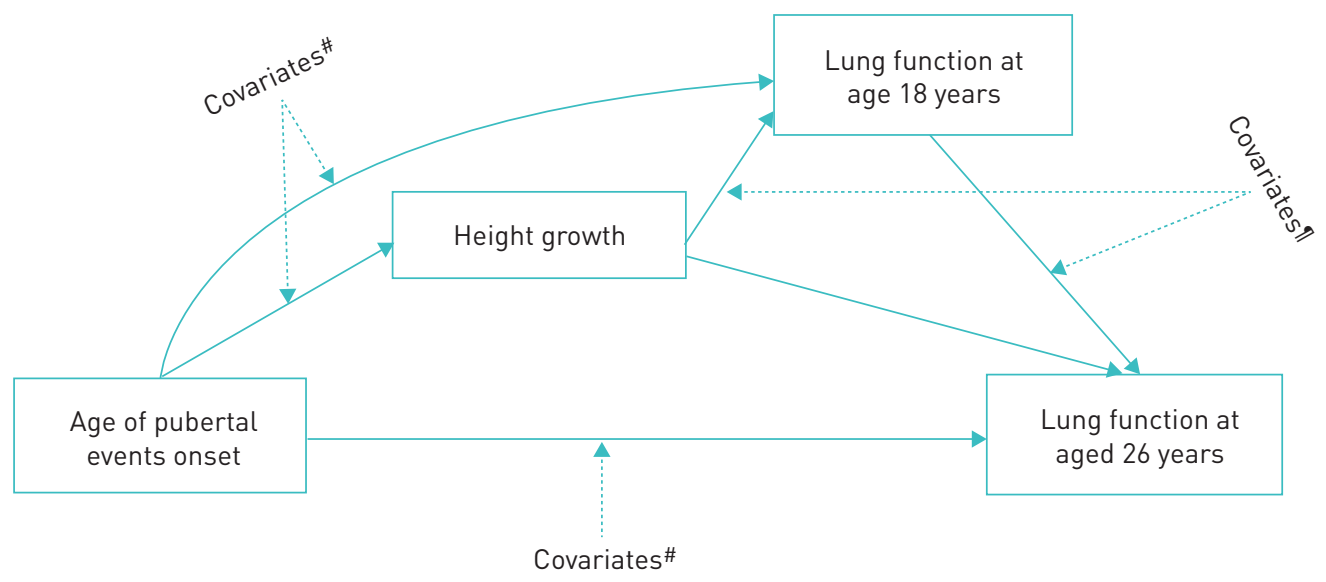

FIGURE 1 The path diagram with two mediators: height growth and lung function at age 18 years. * : asthma status at age 10 years, height at age 10 years, body mass index at age 10 years, socioeconomic status at age 10 years, birth weight status and maternal smoking during pregnancy; ${ }^{\uparrow}$ : smoking at age 18 years. 
was calculated using height measurements at age 10 and 17 years. In total, complete data on 2296 females and 1409 males were included in the analyses. The same path analyses modelling with comparable covariates were applied, and results with $\mathrm{p}$-value $<0.05$ were treated as being statistically significant.

\section{Results}

\section{Characteristics of the study population}

Using one-sample t-tests, we compared the subsamples with complete data on ages of pubertal event onset, lung function measures, and height at ages 10 and 18 years, stratified by sex. No statistically significant differences were identified (all p-values $\geqslant 0.05$, table 1 ).

\section{Mediation analysis in the IOW cohort}

Ages of menarche and body hair growth in females, and body hair growth, growth spurt, voice deepening and facial hair growth in males were included in the path analyses due to their associations with height growth and lung function at ages 18 or 26 years (tables S1-S3 in supplementary material S.2).

For females, significant indirect effects of the age of onset of pubertal events via height growth on the lung function parameters FVC and $\mathrm{FEV}_{1}$ were detected. After adjusting for confounding factors, path analyses indicated that later menarche had an indirect effect on higher FVC (indirect effect coefficient (IEC) $=0.13$; 95\% CI: $0.05,0.20 ; \mathrm{p}=1.28 \times 10^{-3}$ ) (table 2, supplementary figure S2) and $\mathrm{FEV}_{1}$ (IEC=0.09; 95\% CI: 0.01, $0.17 ; \mathrm{p}=0.028$ ) (table 2) via height growth during adolescence and lung function (FVC and $\mathrm{FEV}_{1}$, respectively) at age 18 years. Similar indirect effects were also seen for later age of body hair growth and higher $\mathrm{FVC}$ and $\mathrm{FEV}_{1}$ at age 26 years (FVC: IEC=0.08; 95\% CI: 0.01, 0.15; p=0.017; FEV 1 : IEC=0.07; 95\% CI: $0.01,0.14 ; \mathrm{p}=0.043$ ) (table 2). For these two pubertal events (menarche and body hair growth), no statistically significant direct effects on FVC at age 26 were observed. For $\mathrm{FEV}_{1}$, there was a significant

TABLE 1 Comparison of the analytical subsample $(n=888$ ) with the whole Isle of Wight (IOW) cohort ( $n=1261$ ) with regard to age of onset of pubertal event, lung function and height

\begin{tabular}{|c|c|c|c|}
\hline Variables & Cohort samples & Subsamples & p-value \\
\hline Subjects $n$ & 1261 & 888 & \\
\hline \multicolumn{4}{|l|}{ Age of pubertal events years } \\
\hline \multicolumn{4}{|l|}{ Female } \\
\hline Breast growth & $12.43 \pm 1.58$ & $12.45 \pm 1.58$ & 0.87 \\
\hline Body hair growth & $12.25 \pm 1.43$ & $12.26 \pm 1.41$ & 0.91 \\
\hline Growth spurt & $12.52 \pm 1.70$ & $12.54 \pm 1.70$ & 0.90 \\
\hline Skin changes & $13.11 \pm 1.50$ & $13.06 \pm 1.50$ & 0.55 \\
\hline Menarche & $12.72 \pm 1.42$ & $12.72 \pm 1.37$ & 0.95 \\
\hline \multicolumn{4}{|l|}{ Male } \\
\hline Body hair growth & $13.41 \pm 1.37$ & $13.28 \pm 1.34$ & 0.10 \\
\hline Growth spurt & $13.72 \pm 1.67$ & $13.58 \pm 1.64$ & 0.14 \\
\hline Voice deepening & $14.24 \pm 1.24$ & $14.14 \pm 1.21$ & 0.14 \\
\hline Facial hair growth & $15.38 \pm 1.16$ & $15.28 \pm 1.15$ & 0.11 \\
\hline Skin changes & $13.99 \pm 1.38$ & $13.97 \pm 1.36$ & 0.81 \\
\hline \multicolumn{4}{|l|}{ Lung function age 26 years $L$} \\
\hline \multicolumn{4}{|l|}{ Female } \\
\hline FVC & $4.24 \pm 0.54$ & $4.27 \pm 0.54$ & 0.32 \\
\hline $\mathrm{FEV}_{1}$ & $3.42 \pm 0.43$ & $3.44 \pm 0.42$ & 0.49 \\
\hline \multicolumn{4}{|l|}{ Male } \\
\hline FVC & $5.85 \pm 0.82$ & $5.88 \pm 0.81$ & 0.58 \\
\hline $\mathrm{FEV}_{1}$ & $4.61 \pm 0.72$ & $4.63 \pm 0.71$ & 0.67 \\
\hline \multicolumn{4}{|l|}{ Height $\mathrm{cm}$} \\
\hline \multicolumn{4}{|l|}{ Female } \\
\hline Age 10 & $139.10 \pm 6.47$ & $139.10 \pm 6.40$ & 0.92 \\
\hline Age 18 & $164.70 \pm 6.33$ & $164.80 \pm 6.17$ & 0.78 \\
\hline \multicolumn{4}{|l|}{ Male } \\
\hline Age 10 & $139.00 \pm 5.89$ & $139.10 \pm 5.84$ & 0.74 \\
\hline Age 18 & $178.20 \pm 6.87$ & $177.90 \pm 6.63$ & 0.42 \\
\hline
\end{tabular}

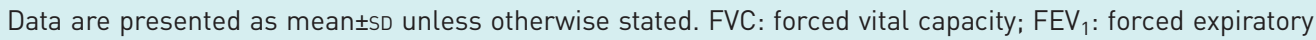
volume in $1 \mathrm{~s}$. 
TABLE 2 Statistically significant effects of age of pubertal events onset on lung function at age 26 years through height growth and lung function at age 18 years in females in the Isle of Wight (IOW) cohort, further tested in the replication cohort, Avon Longitudinal Study of Parents and Children (ALSPAC)

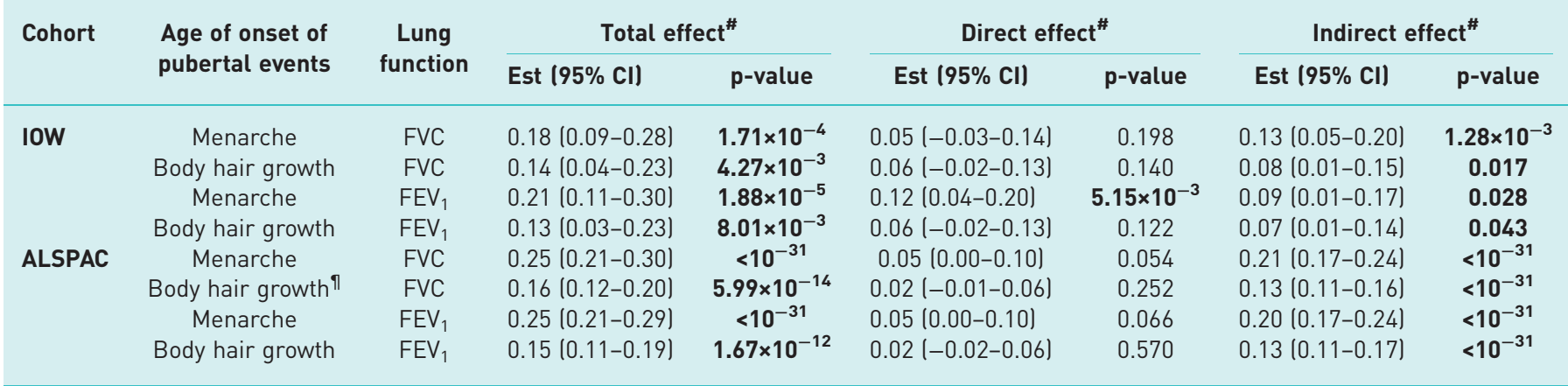

For the IOW, only statistically significant results are included in the table. Est: regression coefficient estimate; FVC: forced vital capacity; FEV forced expiratory volume in $1 \mathrm{~s}$. \#: unit for all the regression coefficients is $L \cdot y e a r^{-1}$ representing expected lung function change for 1 -year increase in pubertal age; ": age at body hair growth is identified by age at attainment of Tanner stage $>2$ in ALSPAC cohort. Bold indicates statistically significant $p$-values.

direct effect of age at menarche on $\mathrm{FEV}_{1}$ at 26 years (direct effect coefficient $(\mathrm{DEC})=0.12 ; 95 \% \mathrm{CI}: 0.04$, $0.20 ; \mathrm{p}=5.15 \times 10^{-3}$ ), in addition to its indirect effects (table 2).

For males, we identified three pubertal events with similar indirect associations with FVC at age 26, as observed in females (table 3), body hair growth (IEC $=0.08 ; 95 \%$ CI: $0.01,0.15 ; \mathrm{p}=0.047$ ), growth spurt (IEC=0.09; 95\% CI: 0.01, 0.17; $\mathrm{p}=0.034$ ), and facial hair growth (IEC $=0.09 ; 95 \% \mathrm{CI}: 0.02,0.16 ; \mathrm{p}=0.014$ ). For example, after adjusting for other covariates in the analyses, later ages of first body hair growth was associated with larger height growth, which was further associated with higher FVC at ages 18 and/or 26 (figure S3). Age of facial hair growth also had a significant direct effect on FVC (DEC $=0.09 ; 95 \%$ CI: 0.01, $0.16 ; \mathrm{p}=0.026$; table 3 ). We did not identify any statistically significant indirect effects for $\mathrm{FEV}_{1}$ in males.

\section{Replication analyses in the ALSPAC cohort}

The statistically significant findings identified in the path analyses in the IOW cohort were further tested in the ALSPAC cohort using lung function measures at ages 15/17 and 24 years. In females, mediation effects of height growth were observed in ALSPAC for all the pubertal events identified in the IOW cohort, with respect to statistical significance as well as directions of association (table 2, Figure S4-S6). Later age at menarche was indirectly associated with higher $\mathrm{FVC}$ and $\mathrm{FEV}_{1}$ at age 24 years via height growth and age $15 / 17$ lung function (for FVC, IEC $=0.21 ; 95 \%$ CI: 0.17, 0.24; $<<10^{-31}$; and for $\mathrm{FEV}_{1}, \mathrm{IEC}=0.20 ; 95 \%$ CI: $0.17,0.24 ; \mathrm{p}<10^{-31}$, respectively). Comparable effects of the age of body hair growth in females were also observed (table 2, Figure S5 and S6). For males, the effects of age at body hair growth were consistent

TABLE 3 Statistically significant effects of age of pubertal events onset on lung function at age 26 years through height growth and lung function at age 18 years in males in the Isle of Wight (IOW) cohort, further tested in the replication cohort, Avon Longitudinal Study of Parents and Children (ALSPAC)

\begin{tabular}{|c|c|c|c|c|c|c|c|c|}
\hline \multirow[t]{2}{*}{ Cohort } & \multirow{2}{*}{$\begin{array}{l}\text { Age of onset of } \\
\text { pubertal events }\end{array}$} & \multirow{2}{*}{$\begin{array}{l}\text { Lung } \\
\text { function }\end{array}$} & \multicolumn{2}{|c|}{ Total effect ${ }^{\#}$} & \multicolumn{2}{|c|}{ Direct effect ${ }^{\#}$} & \multicolumn{2}{|c|}{ Indirect effect } \\
\hline & & & Est $(95 \% \mathrm{CI})$ & p-value & Est $(95 \% \mathrm{CI})$ & p-value & Est $(95 \%$ CI $)$ & p-value \\
\hline \multirow[t]{2}{*}{ IOW } & Body hair growth & FVC & $0.15(0.04-0.25)$ & $5.77 \times 10^{-3}$ & $0.07(-0.01-0.15)$ & 0.078 & $0.08(0.01-0.15)$ & 0.047 \\
\hline & Facial hair growth & FVC & $0.18(0.08-0.28)$ & $5.35 \times 10^{-4}$ & $0.09(0.01-0.16)$ & 0.026 & $0.09(0.02-0.16)$ & 0.014 \\
\hline ALSPAC & Body hair growth" & FVC & $0.07(0.02-0.12)$ & 0.006 & $0.03(-0.02-0.08)$ & 0.219 & $0.04(0.01-0.07)$ & 0.003 \\
\hline
\end{tabular}

For the IOW, only statistically significant results are included in the table. Est: regression coefficient estimate; FVC: forced vital capacity. ${ }^{\#}$ : unit for all the regression coefficients is $L \cdot y e a r^{-1}$ representing expected lung function change for 1 -year increase in pubertal age. ${ }^{1}$ : in the IOW cohort, mediation effects were observed for FVC only. Thus, in the replication cohort ALSPAC, only FVC was evaluated. The ages of growth spurt and facial hair growth were not available in ALSPAC. Age at body hair growth is identified by age at attainment of Tanner stage $>2$ in ALSAPC cohort. Bold indicates statistically significant $p$-values. 
with (but smaller than) those identified in IOW (table 3) with statistically significant indirect effects on FVC (IEC $=0.04 ; 95 \%$ CI: 0.01, 0.07; $\mathrm{p}=0.003$ ).

\section{Discussion}

Using IOW as the discovery cohort and ALSPAC as the replication cohort, this study has demonstrated using path analysis that height growth during adolescence in both sexes mediates the association of age at pubertal onset with lung function parameters FVC in adults. For $\mathrm{FEV}_{1}$, the same pattern as for FVC was observed in females, but not in males.

With respect to the total effects (direct effects plus indirect effects), for females, our results support findings from previous studies that early age at menarche is associated with reduced FVC and $\mathrm{FEV}_{1}$ in young adults $[12,13,15]$. In addition, this study extends these previous observations by demonstrating that the majority of the total effects of age at onset of pubertal events on adult lung function are indirect, via the effect of age at puberty on height growth. The dominance of indirect effects of age at puberty in females highlights the importance of adolescence growth on lung function development. The indirect effects of age at menarche explained $72 \%$ and $84 \%$ of the total effects in the IOW and ALSPAC cohorts, respectively, and thus a much larger sample size was required to detect the remaining small amount of direct effects. The contribution of indirect effects in this study is higher than those observed in a recent study, where $40 \%$ of the total effects of an early age at menarche on FVC at $~ 53$ years was explained by indirect effects via adult-attained height [30]. The discrepancy might have been due to the use of different mediators related to height as well as the age at which FVC is measured. In our study, the mediator height growth took the baseline height into account rather than one time point height, and FVC was measured at a much younger age (26 years), an age with FVC still close to its maximum value, while at age $\sim 53$ years, significant lung function decline was expected.

Putting together the findings in females and males, indirect effects of age of puberty on $\mathrm{FEV}_{1}$ were shown in females but not males. These different relationships might be attributed to a different pattern of lung function development during adolescence in both sexes [17]. Females have a shorter duration of lung function growth during adolescence and attain maximum lung volumes at an earlier age after puberty [15, 31]. At age 18, lung volume growth has almost reached maximum values in females but continues to increase in males until around 20 years [31]. Although further studies are warranted, the findings of both this study and previous studies imply that development of $\mathrm{FVC}$ and $\mathrm{FEV}_{1}$ in adolescence in females are likely to follow similar patterns $[32,33]$, while in males growth of $\mathrm{FVC}$ and $\mathrm{FEV}_{1}$ during adolescence may follow different patterns.

To our knowledge, this is the first study that has examined whether and to what extent height growth during adolescence mediates the effect of age of pubertal onset on lung function longitudinally in both females and males. Our study offers an insight to explore possible "causal pathways" from pubertal onset to lung function in young adulthood [34] and an opportunity to better understand the role of height growth in the connection between pubertal events and lung function. In addition to height growth, peak velocity of height growth, although not available in the IOW cohort, may be another mediator as previously observed in the ALSPAC cohort [15].

This study has some limitations. Age at pubertal events was determined retrospectively based on responses to questionnaires collected at age 18 years in IOW, and recall bias might have affected the reports. However, internal consistency of the age of onset of the different pubertal events in the IOW has previously been demonstrated, implying the validity of these variables [16]. In the ALSPAC cohort, age of onset of some pubertal events was measured by tracking pubertal growth using Tanner stages at follow-ups from 9 to 17 years $[35,36]$, and misclassification of pubertal stages might occur. Finally, in the path analysis, we might have overlooked other unknown confounders that might impact the mediation effects of height growth in adolescence on the association of age at puberty with lung function in early adulthood.

\section{Conclusions}

Our study demonstrated that height growth during adolescence in females mediated the association of age of pubertal onset with $\mathrm{FVC}$ and $\mathrm{FEV}_{1}$ in late adolescence and young adulthood. In males, such mediation effects were identified for FVC but not $\mathrm{FEV}_{1}$, implying dysanaptic growth of FVC and $\mathrm{FEV}_{1}$ during adolescence between the two sexes. The findings indicate the need to promote height growth in adolescence through interventions such as better nutrition and appropriate physical activities to improve lung function in adulthood and reduce future risk of COPD.

Acknowledgements: We are indebted to all participants and their families who were followed up in the Isle of Wight 1989 birth cohort and the Avon Longitudinal Study of Parents and Children cohort over the past two decades. We also appreciate the hard efforts of these two research teams in maintaining the cohorts and collecting data. 
Author contributions: The original concept and design were initiated by Hongmei Zhang and Wilfried Karmaus. Data analyses were performed by Liang $\mathrm{Li}$ and Hongmei Zhang. Data interpretation and manuscript writing were led by Liang Li and Hongmei Zhang. Critical comments on the manuscript and final approval of the manuscript were given by all the authors: Liang Li, Hongmei Zhang, John W. Holloway, A. John Henderson, Susan Ewart, Caroline L. Relton, S. Hasan Arshad and Wilfried Karmaus.

Conflict of interest: None declared.

Support statement: This work was supported by the National Institutes of Health research funds R01AI121226 (MPI: H. Zhang and J.W. Holloway). The UK Medical Research Council and Wellcome (Grant ref: 102215/2/13/2) and the University of Bristol provide core support for ALSPAC. A comprehensive list of grants funding is available on the ALSPAC website (www.bristol.ac.uk/alspac/external/documents/grant-acknowledgements.pdf). The sponsor had no role in study design, data collection and analysis, or the preparation of the manuscript. This publication is the work of the authors and $\mathrm{H}$. Zhang will serve as guarantor for the contents of this paper. Funding information for this article has been deposited with the Crossref Funder Registry.

\section{References}

1 Maestu LP, de Pedro JG. Lung function tests in clinical decision-making. Arch Bronconeumol 2012; 48: 161-169.

2 Baughman P, Marott JL, Lange P, et al. Health outcomes associated with lung function decline and respiratory symptoms and disease in a community cohort. COPD 2011; 8: 103-113.

3 Wedzicha JA. Airway infection accelerates decline of lung function in chronic obstructive pulmonary disease. Am J Respir Crit Care Med 2001; 164: 1757-1758.

$4 \quad$ Young R, Hopkins R. Lung function predicts lung cancer. Eur Respir J 2010; 35: 1421-1422.

5 Ranu H, Wilde M, Madden B. Pulmonary function tests. Ulster Med J 2011; 80: 84-90.

6 Agusti A, Faner R. Lung function trajectories in health and disease. Lancet Respir Med 2019; 7: 358-364.

7 Karmaus W, Mukherjee N, Janjanam VD, et al. Distinctive lung function trajectories from age 10 to 26 years in men and women and associated early life risk factors - a birth cohort study. Respir Res 2019; 20: 98.

8 Bui DS, Lodge CJ, Burgess JA, et al. Childhood predictors of lung function trajectories and future COPD risk: a prospective cohort study from the first to the sixth decade of life. Lancet Respir Med 2018; 6: 535-544.

9 Agusti A, Noell G, Brugada J, et al. Lung function in early adulthood and health in later life: a transgenerational cohort analysis. Lancet Respir Med 2017; 5: 935-945.

10 Vasquez MM, Zhou M, Hu C, et al. Low lung function in young adult life is associated with early mortality. Am J Respir Crit Care Med 2017; 195: 1399-1401.

11 Turner S. Lung function tracking - does it wobble during adolescence? Am J Respir Crit Care Med 2018; 198: $1470-1471$.

12 Macsali F, Real FG, Plana E, et al. Early age at menarche, lung function, and adult asthma. Am J Respir Crit Care Med 2011; 183: 8-14.

13 Gill D, Sheehan NA, Wielscher M, et al. Age at menarche and lung function: a Mendelian randomization study. Eur J Epidemiol 2017; 32: 701-710.

14 Murri V, Antoniazzi F, Piazza M, et al. Lung function in women with idiopathic central precocious puberty: a pilot study. Horm Res Paediatr 2017; 87: 95-102.

15 Mahmoud $\mathrm{O}$, Granell R, Tilling $\mathrm{K}$, et al. Association of height growth in puberty with lung function: a longitudinal study. Am J Respir Crit Care Med 2018; 198: 1539-1548.

16 Yousefi M, Karmaus W, Zhang H, et al. Relationships between age of puberty onset and height at age 18 years in girls and boys. World J Pediatr 2013; 9: 230-238.

17 Neve V, Girard F, Flahault A, et al. Lung and thorax development during adolescence: relationship with pubertal status. Eur Respir J 2002; 20: 1292-1298.

18 DeGroodt EG, van Pelt W, Borsboom GJ, et al. Growth of lung and thorax dimensions during the pubertal growth spurt. Eur Respir J 1988; 1: 102-108.

19 Sherrill DL, Camilli A, Lebowitz MD. On the temporal relationships between lung function and somatic growth. Am Rev Respir Dis 1989; 140: 638-644.

20 Gladysheva ES, Malhotra A, Owens RL. Influencing the decline of lung function in COPD: use of pharmacotherapy. Int J Chron Obstruct Pulmon Dis 2010; 5: 153-164.

21 MacKinnon DP, Fairchild AJ, Fritz MS. Mediation analysis. Annu Rev Psychol 2007; 58: 593-614.

22 Arshad SH, Holloway JW, Karmaus W, et al. Cohort profile: The Isle Of Wight Whole Population Birth Cohort (IOWBC). Int J Epidemiol 2018; 47: 1043-1044i.

23 Graham BL, Steenbruggen I, Miller MR, et al. Standardization of Spirometry 2019 Update. An Official American Thoracic Society and European Respiratory Society Technical Statement. Am J Respir Crit Care Med 2019; 200: e70-e88.

24 Standardization of Spirometry, 1994 Update. American Thoracic Society. Am J Respir Crit Care Med 1995; 152: $1107-1136$.

25 Asher MI, Keil U, Anderson HR, et al. International Study of Asthma and Allergies in Childhood (ISAAC) rationale and methods. Eur Respir J 1995; 8: 483-491.

26 Baron RM, Kenny DA. The moderator-mediator variable distinction in social psychological research: conceptual, strategic, and statistical considerations. J Pers Soc Psychol 1986; 51: 1173-1182.

27 Boyd A, Golding J, Macleod J, et al. Cohort Profile: the 'children of the 90s' - the index offspring of the Avon Longitudinal Study of Parents and Children. Int J Epidemiol 2013; 42: 111-127.

28 Fraser A, Macdonald-Wallis C, Tilling K, et al. Cohort profile: the Avon Longitudinal Study of Parents and Children: ALSPAC mothers cohort. Int J Epidemiol 2013; 42: 97-110.

29 Northstone K, Lewcock M, Groom A, et al. The Avon Longitudinal Study of Parents and Children (ALSPAC): an update on the enrolled sample of index children in 2019. Wellcome Open Res 2019; 4: 51-51.

30 Campbell B, Simpson JA, Bui DS, et al. Early menarche is associated with lower adult lung function: a longitudinal cohort study from the first to sixth decade of life. Respirology (Carlton, Vic) 2020; 25: 289-297. 
31 Kohansal R, Martinez-Camblor P, Agusti A, et al. The natural history of chronic airflow obstruction revisited: an analysis of the Framingham offspring cohort. Am J Respir Crit Care Med 2009; 180: 3-10.

32 Merkus PJ, Borsboom GJ, Van Pelt W, et al. Growth of airways and air spaces in teenagers is related to sex but not to symptoms. J Appl Physiol 1993; 75: 2045-2053.

33 Mosquera RA, Hashmi SS, Pacheco SE, et al. Dysanaptic growth of lung and airway in children with post-infectious bronchiolitis obliterans. Clin Respir J 2014; 8: 63-71.

34 Hafeman DM, Schwartz S. Opening the Black Box: a motivation for the assessment of mediation. Int J Epidemiol 2009; 38: 838-845.

35 Christensen KY, Maisonet M, Rubin C, et al. Pubertal pathways in girls enrolled in a contemporary British cohort. Int J Pediatr 2010; 2010: 329261.

36 Booth JN, Tomporowski PD, Boyle JM, et al. Associations between executive attention and objectively measured physical activity in adolescence: findings from ALSPAC, a UK cohort. Mental Health Phys Act 2013; 6: 212-219. 